\title{
Lichenicolous fungi from Kodar Range, Trans-Baikal Territory of Russia
}

\author{
Mikhail P. Zhurbenko ${ }^{1}$, Sergei V. Chesnokov ${ }^{2} \&$ Ludmila A. Konoreva $^{2,3}$ \\ ${ }^{1}$ Laboratory of the Systematics and Geography of Fungi, Komarov Botanical Institute, Russian Academy of Sciences, \\ Professora Popova str., 2, St. Petersburg, 197376, Russia. \\ E-mail: zhurb58@gmail.com \\ ${ }^{2}$ Laboratory of Lichenology and Bryology, Komarov Botanical Institute, Russian Academy of Sciences, \\ Professora Popova str., 2, St. Petersburg, 197376, Russia. \\ E-mail: lukinbrat@mail.ru \\ ${ }^{3}$ Laboratory of Flora and Vegetation, Polar-Alpine Botanical Garden and Institute, Kirovsk, 184256, Russia. \\ E-mail: ajdarzapov@yandex.ru
}

\begin{abstract}
Sixty three species of lichenicolous and allied fungi are reported, 47 of which are new to Trans-Baikal Territory of Russia. A presumably undescribed Llimoniella-like discomycete on Protoblastenia terricola is illustrated and discussed. Plectocarpon melanohaleae is first recorded in the northern hemisphere. Diplolaeviopsis ranula, Endococcus alectoriae, E. verrucisporus, Epigloea filifera, Sagediopsis fissurisedens and Talpapellis peltigerae var. peltigerae are new to Russia and Asia. Endococcus macrosporus is new to Russia. Lichenostigma rupicolae, Opegrapha geographicola and Unguiculariopsis lettaui are new to Siberia. Arthonia molendoi, Dactylospora glaucomarioides, Didymocyrtis consimilis, Epibryon conductrix, Muellerella lichenicola, Phaeospora cf. rimosicola, Sagediopsis aspiciliae, S. pertusariicola, Sphaerellothecium contextum, Stigmidium conspurcans, S. mitchellii, S. solorinarium and Talpapellis peltigerae are new to South Siberia. Cercidospora thamnoliae and Sagediopsis pertusariicola are first reported outside the Arctic. Athallia is a new host genus for Didymocyrtis consimilis and Bryoria for Endococcus alectoriae. The following new host species are registered: Pertusaria geminipara for Dactylospora deminuta, Calvitimela talayana for Endococcus propinquus s. 1., Bryoria simplicior for Lichenostigma maureri and Melanohalea olivacea for Plectocarpon melanohaleae.
\end{abstract}

Keywords: lichen-inhabiting fungi, biogeography, taxonomy, ecology, Stanovoe Upland, South Siberia

\section{INTRODUCTION}

This paper presents the results of a revision of lichenicolous fungi collected during the study of lichen flora of Kodar Range located in South Siberia. Lichenicolous fungi of this part of Siberia have been specifically treated in several publications (Zhurbenko \& Davydov, 2000; Zhurbenko \& Otnyukova, 2001; Zhurbenko \& Yakovchenko, 2014), but still remain insufficiently revealed, as evidenced by many biogeographic novelties documented in this article.

Kodar is one of the highest ranges of Stanovoe Upland located in South Siberia to the northeast of Baikal Lake (Fig. 1) within $56-58^{\circ} \mathrm{N}$ and $115-119^{\circ} \mathrm{E}$ (Kulakov et al., 2002). Its central peaks mainly rise to the heights of 2700-2800 $\mathrm{m}$. The climate of Kodar is sharply continental with long cold winter and short cool summer. The mean annual air temperature ranges from $-5{ }^{\circ} \mathrm{C}$ below to $-12{ }^{\circ} \mathrm{C}$ above. The mean temperature of the warmest month (July) ranges from $+2{ }^{\circ} \mathrm{C}$ to $+16{ }^{\circ} \mathrm{C}$, while the mean temperature of the coldest month (January) ranges from $-30{ }^{\circ} \mathrm{C}$ to $-40{ }^{\circ} \mathrm{C}$. The mean annual precipitation is 350-1200 $\mathrm{mm}$. The vegetation strongly reflects a vertical zonation caused by the mountainous terrain and forms three altitudinal belts. Forest belt is mainly dominated by Larix gmelinii, the other common trees are Abies sibirica, Betula platyphylla, B. lanata, Chosenia arbutifolia, Picea obovata, Pinus sibirica, P. sylvestris,

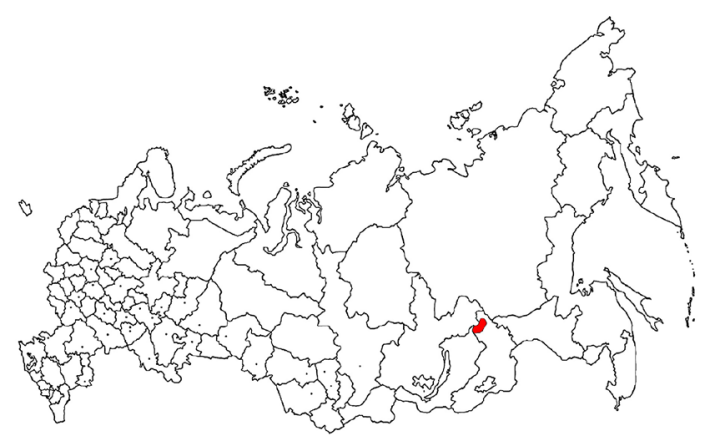

Fig. 1. Location of the study area in Russia. 
Populus suaveolens and P. tremula. Subalpine belt is mainly composed of sparse Larix gmelinii forests and Pinus pumila, Betula divaricata and Rhododendron aureum shrubs interspersed with Duschekia fruticosa and Salix spp. thickets. Alpine belt is dominated by lichen tundras combined with a patchy plant cover and stone fields mostly inhabited by crustaceous lichens.

\section{MATERIAL AND METHODS}

The study is based on 124 specimens of lichenicolous fungi which are deposited in LE and VLA (one specimen) herbaria. The material was examined with standard microscopic techniques using dissecting microscope Stemi 2000-CS and compound microscope Axio Imager A1 equipped with Nomarski differential interference contrast optics (DIC). Microscopic examination was done in water, $10 \% \mathrm{KOH}(\mathrm{K})$, Lugol's iodine, directly (I) or after a $\mathrm{KOH}$ pre-treatment $(\mathrm{K} / \mathrm{I})$, or Brilliant Cresyl blue (BCr). Measurements were taken from water mounts, unless otherwise indicated. The length, breadth and length/breadth ratio $(1 / b)$ of ascospores and conidia are given (where $\mathrm{n}>10)$ as (minimum-) $\{\mathrm{X}-\mathrm{SD}\}-\{\mathrm{X}+\mathrm{SD}\}(-\operatorname{maxi}-$ mum), where $X$ is the arithmetic mean, and SD the corresponding standard deviation, followed by the number of measurements.

\section{THE SPECIES}

All collections are from Kodar Range of Stanovoe Upland in South Siberia administratively belonging to the Kalarskii District of Trans-Baikal Territory of Russia. Species new to Trans-Baikal Territory are denoted by an asterisk (*).

Abrothallus bertianus De Not. s. 1. - Anarga River, 56 55'11" N, 11801'45" E, elev. $941 \mathrm{~m}$, mixed forest, on Melanelixia glabratula (thallus), 9.07.2013, L. A. Konoreva (LE 309219); same river, 56 55'19" N, 118 01'50" E, elev. $1071 \mathrm{~m}$, mixed forest, on Melanohalea olivacea (apothecia, thallus), 9.07.2013, Yu. V. Gerasimova (LE 309351a; teleomorph and anamorph).

Notes - Apothecia subglobose and somewhat applanate, constricted at the base, up to 400 $\mu \mathrm{m}$ diam., sometimes slightly pruinose. Epihymenium medium brown to partly purple, with large orange granules dissolving in $\mathrm{K}, \mathrm{K}+$ olive green. Hymenium colourless to partly purple, $\mathrm{K}+$ greenish. Hypothecium medium gray brown/ brown, $\mathrm{K}+$ olive. Ascospores pale to medium olive gray/yellowish brown, distinctly verrucose, $(11.2-) 11.5-14.9(-19.5) \times(4.1-) 4.7-5.9(-7.7) \mu \mathrm{m}$, $1 / \mathrm{b}=(2.1-) 2.2-2.8(-3.5)(\mathrm{n}=40, \mathrm{LE} 309219)$ or (11.7-)12.6-15.6(-19.5) × (4.6-)4.9-6.3(-8.5) $\mu \mathrm{m}$, $1 / \mathrm{b}=(1.9-) 2.1-2.9(-3.5)(\mathrm{n}=71, \mathrm{LE} 309351 \mathrm{a})$, (0-)1-septate, not to occasionally markedly constricted at the septum, occasionally with halo ca. $1 \mu \mathrm{m}$ thick (better seen in $\mathrm{K}$ ). Conidia $(7.7-) 8.4-11.4(-12.8) \times(3.6-) 3.9-4.7(-5.0) \mu \mathrm{m}$, $1 / \mathrm{b}=(1.5-) 1.9-2.9(-3.6)(\mathrm{n}=25$, observed only in LE 309351a).

The type of Abrothallus bertianus is confined to species of Melanelixia (Lawrey \& Diederich, 2016; P. Diederich, pers. comm.), however, the fungus has also been reported from species of Melanohalea (Santesson, 1960; Hafellner \& Wieser, 2000; Suija, 2005; Alstrup \& Ahti, 2007; Brackel, 2011). It is noteworthy that the examined specimens on Melanelixia and Melanohalea clearly differ in size of their ascospores.

*ABRothallus PaRmeliarum (Sommerf.) Arnold Shan'go River, 56 ${ }^{\circ} 7^{\prime} 31^{\prime \prime}$ N, $117^{\circ} 48^{\prime} 13^{\prime \prime}$ E, elev. $1715 \mathrm{~m}$, subalpine Larix gmelinii thin forest, on Parmelia omphalodes (thallus), 9.06.2014, S. V. Chesnokov (LE 309220); Syul'ban River, 56 50'41" N, 117²17'57" E, elev. $1381 \mathrm{~m}$, Chosenia arbutifolia forest, on $P$. sulcata (thallus), 13.06.2015, S. V. Chesnokov (LE 309322a); $56^{\circ} 40^{\prime} 12^{\prime \prime} \mathrm{N}, 117^{\circ} 10^{\prime} 38^{\prime \prime} \mathrm{E}$, elev. $1054 \mathrm{~m}$, mixed forest, on P. sulcata (thallus), 23.06.2015, S. V. Chesnokov (LE 309343).

ABROTHAllus PEYRITSCHII (Stein) Kotte - Anarga River, 56 55'19" N, $118^{\circ} 01^{\prime} 50^{\prime \prime}$ E, elev. 1071 $\mathrm{m}$, mixed forest, on Vulpicida pinastri (thallus), 9.07.2013, Yu. V. Gerasimova (LE 309250c; anamorph); Syul'ban River, 56 40'12" N, $117^{\circ} 10^{\prime} 38^{\prime \prime} \mathrm{E}$, elev. $1054 \mathrm{~m}$, mixed forest, on $V$. pinastri (thallus), 23.06.2015, S. V. Chesnokov (LE 309217).

Notes - Diederich (2004) characterized the hymenium of the species as hyaline to pale brownish, pale green in the upper part, but in the examined specimen it is purple. Conidia (5.4-)5.7-7.1(-8.5) × (4.3-)4.4-5.2(-6.5) $\mu \mathrm{m}, 1 / \mathrm{b}$ $=1.2-1.4(-1.7)(\mathrm{n}=35)$.

*Arthonia clemens (Tul.) Th. Fr. - Shan'go Lake, 5658'41" N, 11747'50" E, elev. 1908 m, mountain tundra, on Rhizoplaca chrysoleuca (apothecial discs), 7.06.2014, S. V. Chesnokov (LE 309242); headwaters of Shan'go River, 
5658'34" N, 11749'12" E, elev. 1992 m, mountain tundra, on $R$. chrysoleuca (discs of apothecia), 8.06.2014, L. A. Konoreva (LE 309329).

*ARthonia digitatae Hafellner - Uglovoi Creek, 5656'39" N, $117^{\circ} 36^{\prime} 44^{\prime \prime}$ E, elev. $1691 \mathrm{~m}$, mountain tundra, on Cladonia pyxidata (basal squamules), 16.06.2014, L. A. Konoreva (LE 309328).

*ARTHonia Molendoi (Frauenf.) R. Sant. - Olenii

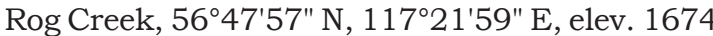
m, subalpine Pinus pumila shrubs, on Polycauliona polycarpa (apothecia, thallus), 18.05.2015, L. A. Konoreva (LE 309334).

Note - New to South Siberia.

Arthonia stereocaulina (Ohlert) R. Sant. Mramornoe gorge, Mramornyi settlement, 5654'38" N, 117²4'29" E, elev. 1806 m, stone field, on Stereocaulon sp. (phyllocladia, apothecia), 3.07.2013, L. A. Konoreva (LE 309358a); Surpriznoe Lake, 56 $54^{\prime} 21^{\prime \prime} \mathrm{N}, 117^{\circ} 38^{\prime} 20^{\prime \prime}$ E, elev. $2011 \mathrm{~m}$, mountain tundra, on S. vesuvia num (phyllocladia), 5.07.2013, L. A. Konoreva (LE 309276b); headwaters of Shan'go River, 56 58'39" N, 117 49'08" E, elev. 2063 m, boulder field, on S. botryosum (phyllocladia), 8.06.2014, S. V. Chesnokov (LE 309355a); Shan'go River, $56^{\circ} 57^{\prime} 31^{\prime \prime} \mathrm{N}, 117^{\circ} 48^{\prime} 13^{\prime \prime} \mathrm{E}$, elev. $1715 \mathrm{~m}$, subalpine Larix gmelinii thin forest, on Stereocaulon sp. (phyllocladia), 9.06.2014, L. A. Konoreva (LE 309325); Azarova glacier, 5653'58" N, 117³4'59" E, elev. 2053 m, mountain tundra, on S. symphycheilum (phyllocladia, stems), 13.06.2014, S. V. Chesnokov (LE 309362); confluence of Uglovoi Creek and Srednii Sakukan River, 5656'33" N, 117³6'51" E, elev. 1670 m, mountain tundra, on Stereocaulon sp. (phyllocladia), 15.06.2014, S. V. Chesnokov (LE 309218b); confluence of Syul'ban River and Zolotoi Creek, 56 50'12" N, 117²17'23" E, elev. $1627 \mathrm{~m}$, subalpine Pinus pumila shrubs, on S. rivulorum (phyllocladia), 14.06.2015, S. V. Chesnokov (LE 309216a).

*Carbonea vitellinaria (Nyl.) Hertel - Uglovoe Lake, 5656'52" N, 117³5'43" E, elev. 1892 $\mathrm{m}$, mountain tundra, on Candelariella vitellina (thallus), 17.06.2014, S. V. Chesnokov (VLA).

${ }^{*}$ CAtillaria stereocaulorum (Th. Fr.) H. Olivier Leprindinskoe Plateau, 56 ${ }^{\circ} 40^{\prime} 05^{\prime \prime}$ N, $117^{\circ} 24^{\prime} 50^{\prime \prime}$ E, elev. $2142 \mathrm{~m}$, mountain tundra, on Stereocaulon rivulorum (phyllocladia), 15.08.2012, L. A. Konoreva (LE 309232); Zolotoi Creek,
5655'57" N, 117³6'51" E, elev. 1857 m, rocks in mountain tundra, on S. lambii (phyllocladia), 6.07.2013, M. P. Andreev (LE 309357); Azarova glacier, 5653'58" N, 117³4'59" E, elev. 2053 m, mountain tundra, on $S$. rivulorum (phyllocladia), 13.06.2014, S. V. Chesnokov (LE 309344); Srednii Sakukan River, 5656'28" N, 117 ${ }^{\circ} 36^{\prime} 55^{\prime \prime}$ E, elev. 1686 m, subalpine Salix shrubs, on S. rivulorum (phyllocladia), 18.06.2014, S. V. Chesnokov (LE 309235); Syul'ban River, 56 53'17" $\mathrm{N}, 117^{\circ} 18^{\prime} 48^{\prime \prime} \mathrm{E}$, elev. $1562 \mathrm{~m}$, subalpine Larix gmelinii thin forest, on $S$. cf. subcoralloides (phyllocladia), 10.06.2015, S. V. Chesnokov (LE 309365); confluence of Syul'ban River and Zolotoi Creek, 56 50'12" N, $117^{\circ} 17^{\prime} 25^{\prime \prime}$ E, elev. $1595 \mathrm{~m}$, subalpine Pinus pumila shrubs, on S. rivulorum (phyllocladia), 14.06.2015, S. V. Chesnokov (LE 309233, LE 309216b); Olenii Rog Creek, 5647'57" N, $117^{\circ} 21^{\prime} 59^{\prime \prime}$ E, elev. 1674 m, subalpine Pinus pumila shrubs, on S. rivulorum (phyllocladia), 18.06.2015, S. V. Chesnokov (LE 309234).

*Cercidospora punctillata (Nyl.) R. Sant. - Uglovoe Lake, 5656'41" N, 117 ${ }^{\circ} 35^{\prime} 12^{\prime \prime}$ E, elev. 1918 $\mathrm{m}$, mountain tundra, on Solorina crocea (moribund thallus), 17.06.2014, S. V. Chesnokov (LE 309321b).

Cercidospora stereocaulorum (Arnold) Hafellner - Leprindinskoe Plateau, 56 $40^{\prime} 00^{\prime \prime} \mathrm{N}$, $117^{\circ} 24^{\prime} 52 "$ E, elev. $2042 \mathrm{~m}$, mountain tundra, on Stereocaulon condensatum (cephalodia, phyllocladia, stems), 15.08.2012, M. P. Andreev (LE 309364); Medvezhii Creek, 5654'35" N, $117^{\circ} 36^{\prime} 42^{\prime \prime}$ E, elev. $1796 \mathrm{~m}$, stone field, on $S$. vesuvianum (phyllocladia), 1.07.2013, M. P. Andreev (LE 309354); Zolotoi Creek, 56 56'05" $\mathrm{N}, 117^{\circ} 37^{\prime} 18^{\prime \prime} \mathrm{E}$, elev. $1680 \mathrm{~m}$, mountain tundra, on S. glareosum (cephalodia, phyllocladia, stems), 6.07.2013, M. P. Andreev (LE 309361); same creek, 56 55'57" N, 117 36'51" E, elev. $1857 \mathrm{~m}$, mountain tundra, on S. symphycheilum (phyllocladia), 6.07.2013, L. A. Konoreva (LE 309360); Shan'go River, 5657'31" N, 117²48'13" E, elev. $1715 \mathrm{~m}$, subalpine Larix gmelinii thin forest, on S. condensatum (phyllocladia), 9.06.2014, L. A. Konoreva (LE 309331a); Azarova glacier, 5653'58" N, 117³4'59" E, elev. 2053 $\mathrm{m}$, mountain tundra, on $S$. lambii (phyllocladia), 13.06.2014, S. V. Chesnokov (LE 309356a); Uglovoi Creek, 5656'55" N, 117³6'12" E, elev. $1735 \mathrm{~m}$, rocks, on S. subcoralloides (phyllocladia), 17.06.2014, L. A. Konoreva (LE 309363). 
*Cercidospora thamnoliae Zhurb. - Baltiiskoe gorge, 5654'20" N, 117³9'21" E, elev. 1903 $\mathrm{m}$, mountain tundra, on Thamnolia vermicularis (thallus), 2.07.2013, L. A. Konoreva (LE 309222).

Note - Formerly known only from the Arctic (Zhurbenko, 2012).

*Dactylospora amygdalariae Triebel - Uglovoi Creek, 5656'39" N, $117^{\circ} 36^{\prime} 44^{\prime \prime}$ E, elev. 1691 $\mathrm{m}$, mountain tundra, on Amygdalaria panaeola (cephalodia, thallus), 16.06.2014, L. A. Konoreva (LE 309316); Olenii Rog Creek, 5647'41" N, $117^{\circ} 22^{\prime} 17^{\prime \prime}$ E, elev. $1888 \mathrm{~m}$, mountain tundra, on A. elegantior (thallus), 18.06.2015, S. V. Chesnokov (LE 309342).

*Dactylospora deminuta (Th. Fr.) Triebel -

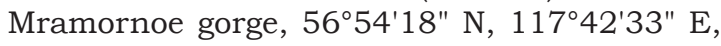
elev. 1885 m, mountain tundra, on Pertusaria geminipara (thallus), 3.07.2013, L. A. Konoreva (LE 309228); confluence of Uglovoi Creek and Srednii Sakukan River, 5656'31" N, 117 $36^{\circ} 6^{\prime} 50^{\prime \prime}$ E, elev. $1690 \mathrm{~m}$, subalpine Salix shrubs, on Biatora cf. vernalis (thallus), 15.06.2014, L. A. Konoreva (LE 309339); headwaters of Olenii

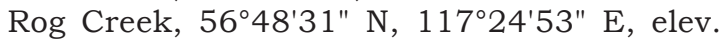
1971 m, mountain tundra, on Pertusaria geminipara (thallus), 16.06.2015, L. A. Konoreva (LE 309243).

Notes - Hymenium hyaline to light reddish brown, exciple and hypothecium strongly reddish brown to brown, violet blue granules not observed. Ascospores (12-)14.1-20.1(-24.0) × (4.5-)5.1-6.3(-7.0) $\mu \mathrm{m}, 1 / \mathrm{b}=(2.0-) 2.4-3.6(-4.8)$ (n = 39), with (1-)3(-7) transsepta.

Pertusaria geminipara is a new host species.

*DACTYLOSPORA GLAUCOMARIOIDES (Tuck.) Hafellner - Olenii Rog Creek, 56²8'13" N, 117²4'16" E, elev. $1827 \mathrm{~m}$, mountain tundra, on Ochrolechia frigida (apothecia, thallus), 16.06.2015, L. A. Konoreva (LE 309229).

Note - New to South Siberia.

*DACTYLOSPORA PERTUSARIICOLA (Tuck.) Hafellner Leprindinskoe Plateau, 56³9'27" N, 117 ${ }^{\circ} 25^{\prime} 34^{\prime \prime}$ E, elev. $1698 \mathrm{~m}$, stone rubble, on Pertusaria aspergilla (thallus), 14.08.2012, L. A. Konoreva (LE 309237); confluence of Syul'ban River and Zolotoi Creek, 56 50'12" N, 117 17'21" E, elev. $1655 \mathrm{~m}$, subalpine Pinus pumila shrubs, on $P$. aspergilla (apothecial margins, thallus), 14.06.2015, S. V. Chesnokov (LE 309267a); headwaters of Olenii Rog Creek, 56 $48^{\prime} 20^{\prime \prime}$ N, $117^{\circ} 25^{\prime} 17^{\prime \prime}$ E, elev. $2020 \mathrm{~m}$, mountain tundra, on $P$. aspergilla (apothecial margins, thallus), 17.06.2015, S. V. Chesnokov (LE 309263a).

Notes - Apothecia up to $0.7 \mathrm{~mm}$ diam. Epihymenium medium brown above, pale olive gray below, hymenium hyaline, subhymenium pale olive gray brown, hypothecium and exciple medium to dark brown; olive gray tinge fading in $\mathrm{K}$. Ascospores ellipsoid to oblong, ends usually rounded, medium brown when mature, (7.5) $8.8-11.8(-14.7) \times(4.4-) 5.0-6.0(-7.0) \mu \mathrm{m}, 1 / \mathrm{b}$ $=(1.4-) 1.6-2.2(-2.6)(\mathrm{n}=67),(0-) 1(-3)$-septate, usually constricted at the septa, smooth-walled, non-halonate.

*Didymocyrtis Consimilis Vain. s. 1. - Shan'go River, 5657'30" N, 11748'20" E, elev. 1720 m, subalpine Larix gmelinii thin forest, on Athallia holocarpa (apothecial discs), 9.06.2014, L. A. Konoreva (LE 309335).

Notes - Ascospores pale brown, narrowly obovoid, with larger upper cell, sometimes markedly so, (9.3-)10.4-13.4(-15.3) × (4.8-)5.2$6.2(-6.3) \mu \mathrm{m}, 1 / \mathrm{b}=(1.6-) 1.8-2.4(-2.9)(\mathrm{n}=28)$, (0-)1(-2)-septate, darkened triangle at the point of contact of the septum and lateral wall sometimes distinct, but not protruding externally, somewhat constricted at the median septum, finely verruculose $(\times 1000$, DIC), halo not seen, overlappingly uniseriate in the ascus.

So far Didymocyrtis consimilis was known with certainty only on Caloplaca cerina group, however, the examined material on Athallia also fits its modern description (Ertz et al., 2015). The species was formerly known in Russia (mostly under its synonym Phoma caloplacae D. Hawksw.) from Karelia Republic (Räsänen, 1939), Bashkortostan Republic (Urbanavichus \& Urbanavichene, 2011), Krasnoyarsk Territory (Hawksworth, 1981) and Chukotka (Zhurbenko, 2009a). New to South Siberia.

*Diplolaeviopsis RanUla Giralt \& D. Hawksw. (asexual stage) - Anarga River, 56 55'19" N, $118^{\circ} 01^{\prime} 50^{\prime \prime} \mathrm{E}$, elev. $1071 \mathrm{~m}$, mixed forest, on Lecanora cf. symmicta (apothecial discs), 9.07.2013, Yu. V. Gerasimova (LE 309250a).

Note - New to Russia and Asia.

*Endococcus alectoriae (D. Hawksw.) D. Hawksw. - Headwaters of Srednii Sakukan River, 5657'59" N, 117³7'60" E, elev. 1925 m, mountain tundra, on Bryoria nitidula (thallus), 16.06.2014, L. A. Konoreva (LE 309353). 
Notes - Ascomata 75-100 $\mu \mathrm{m}$ diam., semiimmersed. Asci 58-63 × 10-14 $\mu \mathrm{m}$. Ascospores shortly clavate/narrowly obovoid, occasionally ellipsoid, usually with rather acute upper and sometimes lower ends, finally medium brown, $(8.3-) 10.0-12.0(-14.0) \times(3.7-) 4.0-4.6(-4.8) \mu \mathrm{m}$, $1 / \mathrm{b}=(1.8-) 2.3-2.9(-3.4)(\mathrm{n}=60),(0-) 1$-septate, slightly constricted at the septum, distinctly granulate, usually with $1(-2)$ large guttules in each cell.

In the species protologue asci were reported as being mainly $50 \times 12 \mu \mathrm{m}$ and ascospores as 10.5-15.5 × 3.5-4.5 um (Hawksworth, 1971). So far Endococcus alectoriae was known only from the type collection in the Austrian Alps growing on Alectoria ochroleuca. Bryoria is a new host genus. New to Russia and Asia.

*Endococcus macrosporus (Arnold) Nyl. - Baltiiskoe gorge, $56^{\circ} 54^{\prime} 20^{\prime \prime} \mathrm{N}, 117^{\circ} 39^{\prime} 21^{\prime \prime} \mathrm{E}$, elev. $1903 m$, mountain tundra, on Rhizocarpon geographicum (thallus), 2.07.2013, M. P. Andreev (LE 309275); Surpriznoe Lake, 56 54'20.5"N, 117³8'20.3"E, elev. $2011 \mathrm{~m}$, mountain tundra, on R. geographicum (thallus), 05.07.2013, L. A. Konoreva (LE 309265); confluence of Syul'ban River and Zolotoi Creek, 56 50'11.9" N, $117^{\circ} 17^{\prime} 21.1^{\prime \prime}$ E, elev. 1655 m, subalpine Pinus pumila shrubs, on $R$. geographicum (thallus), 14.06.2015, S. V. Chesnokov (LE 309267b); headwaters of Olenii Rog Creek, 56 48'19.6" N, $117^{\circ} 25^{\prime} 17.3^{\prime \prime}$ E, elev. $2020 \mathrm{~m}$, mountain tundra, on R. geographicum (thallus), 17.06.2015, S. V. Chesnokov (LE 309263b).

Notes - Ascomata up to $200 \mu \mathrm{m}$ diam., protruding in the upper part. Ascospores ellipsoid to obovoid (wider above), usually with more or less acute apices, long remaining pale to medium olive gray, finally medium brown, darker at the apices and around the septum, (0-)1-sepate, occasionally constricted at the septum, (9.8) 15.3-18.9(-23.2) × (5.8-)6.7-7.9(-9.5) $\mu \mathrm{m}, 1 / \mathrm{b}$ $=(1.4-) 2.1-2.7(-3.6)(\mathrm{n}=201)$.

The species concept accepted here follows Sérusiaux et al. (1999). New to Russia.

*Endococcus PRopinquUs (Körb.) D. Hawksw. s.

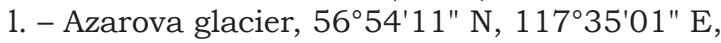
elev. 2020 m, mountain tundra, on Porpidia cf. flavicunda (thallus), 1.07.2013, L. A. Konoreva (LE 309277); Surpriznoe Lake, 5654'21" N, $117^{\circ} 38^{\prime 2} 20^{\prime \prime}$ E, elev. $2011 \mathrm{~m}$, mountain tundra, on P. flavicunda (thallus), 5.07.2013, L. A. Ko- noreva (LE 309271); Zolotoi Creek, 5650'22" N, $117^{\circ} 19^{\prime} 44^{\prime \prime} \mathrm{E}$, elev. $1666 \mathrm{~m}$, mountain tundra, on Calvitimela talayana (thallus), 12.06.2015, L. A. Konoreva (LE 309262); headwaters of Olenii Rog Creek, 56 48'20" N, 117²5'17" E, elev. 2020 $\mathrm{m}$, mountain tundra, on C. armeniaca (thallus), 17.06.2015, S. V. Chesnokov (LE 309338b).

Notes - The species has been described from Porpidia tuberculosa and thereafter reported from various genera of saxicolous crustose lichens including Calvitimela (Triebel, 1989; Santesson et al., 2004). We have not observed distinct differences between the examined specimens on Porpidia and Calvitimela including their ascospore size [(8.7-) 10.9-12.5(-15.8) $\times$ (5.8-)6.4-7.6(-9.5) $\mu \mathrm{m}, 1 / \mathrm{b}=(1.3-) 1.4-1.8(-2.4)$ $(\mathrm{n}=112$, on Porpidia) and (9.0-)9.9-12.3(-14.8) $\times$ (5.5-)6.0-7.0(-7.5) $\mu \mathrm{m}, 1 / \mathrm{b}=(1.3-) 1.5-1.9(-2.2)$ ( $\mathrm{n}=54$, on Calvitimela)] and thus suggest they are conspecific. Calvitimela talayana is a new host species.

Endococcus Rugulosus Nyl. s. 1. - Azarova gla-

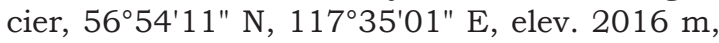
mountain tundra, on Rhizocarpon subgeminatum (thallus), 13.06.2014, L. A. Konoreva (LE 309268); on Aspicilia sp. (thallus), 13.06.2014, S. V. Chesnokov (LE 309244).

Notes - The species was described from Verrucaria macrostoma and subsequently reported from various genera of saxicolous crustose lichens including Aspicilia and Rhizocarpon (Triebel, 1989; Hafellner, 1993; Hafellner \& Türk, 1995). Examined material fits the broad species concept sensu Triebel (1989) including the ascospore sizes, which are (12-)13-16(-16.5) $\times(5.5-) 6-7.5(-8) \mu \mathrm{m}$ according to Triebel (1989), $(12.0-) 12.9-15.7(-16.8) \times(5.8-) 6.2-7.8(-8.4) \mu \mathrm{m}$, $1 / \mathrm{b}=(1.5-) 1.8-2.4(-2.7)(\mathrm{n}=25)$ in the specimen on Aspicilia and (11.3-)12.8-15.8(-17.5) $\times$ (5.1-)5.9-7.7(-8.4) $\mu \mathrm{m}, 1 / \mathrm{b}=(1.6-) 1.7-2.5(-3.1)$ $(\mathrm{n}=46)$ in the specimen on Rhizocarpon.

*Endococcus VERRUCISPORUs Alstrup - Azarova glacier, $56^{\circ} 54^{\prime} 11^{\prime \prime} \mathrm{N}, 117^{\circ} 35^{\prime} 01^{\prime \prime} \mathrm{E}$, elev. 2020 $\mathrm{m}$, mountain tundra, on Ionaspis lacustris (thallus), 13.06.2014, S. V. Chesnokov (LE 309347a). Notes - Ascospores of the examined material are somewhat longer than given in the species protologue, viz. (9.1-)10.5-13.1(-14.4) $\times$ (4.9-)5.8-6.8(-7.4) $\mu \mathrm{m}, 1 / \mathrm{b}=(1.3-) 1.6-2.2(-2.4)$ $(\mathrm{n}=27)$ vs. $10-12 \times 6.5-7.5 \mu \mathrm{m}$ (Alstrup et al., 1994). The species was previously reported from 
Europe and North America, always on Ionaspis lacustris (Sérusiaux et al., 1999; Orange, 2002; Santesson et al., 2004; Kocourková \& van den Boom, 2005; Alstrup et al., 2009; Brackel, 2010). New to Russia and Asia.

*EPIBRYon CONDUCTRIX (Norman) Nik. Hoffm. \& Hafellner - Confluence of Uglovoi Creek and Srednii Sakukan River, 56 56'33" N, 117 ${ }^{\circ} 36^{\prime} 51^{\prime \prime}$ $\mathrm{E}$, elev. $1670 \mathrm{~m}$, mountain tundra, on squamules of a Catapyrenium-like lichen growing on soil, 15.06.2014, S. V. Chesnokov (LE 309218a). Note - New to South Siberia.

Epicladonia sandstedei (Zopf) D. Hawksw. Shan'go River, 5656'22" N, 11748'34" E, elev. $1710 \mathrm{~m}$, mixed forest, on Cladonia cf. pyxidata (basal squamules), 10.06.2014, S. V. Chesnokov (LE 309324); Medvezhii Creek, 5654'46" N, $117^{\circ} 37^{\prime} 05^{\prime \prime}$ E, elev. $1752 \mathrm{~m}$, mountain tundra, on C. cf. pyxidata (basal squamules, podetia), 14.06.2014, S. V. Chesnokov (LE 309348).

*Epigloea filifera Döbbeler - Confluence of Syul'ban River and Zolotoi Creek, 56 50'12" N, $117^{\circ} 17^{\prime} 23^{\prime \prime}$ E, elev. 1627 m, subalpine Pinus pumila shrubs, over Stereocaulon sp. (cephalodia, phyllocladia) close to soil, 14.06.2015, S. V. Chesnokov (LE 309226).

Notes - The species is not truly lichenicolous, but is often listed along with lichenicolous fungi. So far it was reported only from Europe, viz. England, Netherlands, Germany, Austria, Belgium, France and Ukraine (Döbbeler, 1984; Berger et al., 1998; Boissiere \& Montavont, 1998; van den Boom, 1998; Sérusiaux et al., 1999; Khodosovtsev, 2005; Brackel, 2009; Smith et al., 2009). New to Russia and Asia.

*Epigloea soleiformis Döbbeler - Azarova glacier, 5653'58" N, 117 34'59" E, elev. 2053 m, moraine in alpine belt, over Stereocaulon lambii (base of stems), 13.06.2014, S. V. Chesnokov (LE 309356c); Srednii Sakukan River, 5656'28" N, 117³6'55" E, elev. 1686 m, subalpine Salix shrubs, over S. botryosum (stems and phyllocladia near soil), 18.06.2014, S. V. Chesnokov (LE 309366).

Note - The species is not truly lichenicolous, but is often listed along with lichenicolous fungi.

*Lasiosphaeriopsis stereocaulicola (Linds.) O.E. Erikss. \& R. Sant. - Mramornoe gorge, Mramornyi settlement, 56 54'38" N, 117 ${ }^{\circ} 42^{\prime} 29^{\prime \prime}$ E, elev. $1806 \mathrm{~m}$, stone field, on Stereocau- lon sp. (stems), 3.07.2013, L. A. Konoreva (LE 309358b); headwaters of Shan'go River, 5658'39" N, 11749'08" E, elev. 2063 m, boulder field, on S. botryosum (phyllocladia, stems), 8.06.2014, S. V. Chesnokov (LE 309355b); confluence of Uglovoi Creek and Srednii Sakukan River, 5656'33" N, 117³6'51" E, elev. 1670 m, mountain tundra, on Stereocaulon sp. (stems, phyllocladia), 15.06.2014, S. V. Chesnokov (LE 309218c).

Lichenoconium usneae (Anzi) D. Hawksw. -

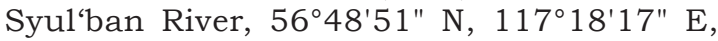
elev. $1341 \mathrm{~m}$, Chosenia arbutifolia forest, on Melanohalea septentrionalis (apothecial discs), 8.06.2015, S. V. Chesnokov (LE 309350).

*Lichenodiplis lecanorae (Vouaux) Dyko \& D. Hawksw. - Syul'ban River, 56 38'02" N, $117^{\circ} 11^{\prime} 51^{\prime \prime}$ E, elev. 1020 m, Larix gmelinii forest, on Evernia mesomorpha (thallus), 23.06.2015, S. V. Chesnokov (LE 309259b).

*Lichenostigma alpinum (R. Sant., Alstrup \& D. Hawksw.) Ertz \& Diederich - Syul'ban River, 56 50'38" N, $117^{\circ} 18^{\prime} 05^{\prime \prime}$ E, elev. 1378 m, Larix gmelinii forest, on Ochrolechia sp. (thallus) growing on lignum, 13.06.2015, S. V. Chesnokov (LE 309349b).

LiChenostigma MAURERI Hafellner - Shan'go River, 5656'20" N, 11748'28" E, elev. $1661 \mathrm{~m}$, Larix gmelinii forest, on Bryoria simplicior (thallus), 10.06.2014, L. A. Konoreva (LE 309314); Syul'ban River, 56 48'51" N, 117 $18^{\circ} 17^{\prime \prime}$ E, elev. $1341 \mathrm{~m}$, Chosenia arbutifolia forest, on Evernia esorediosa (thallus), 8.06.2015, S. V. Chesnokov (LE 309315b); same river, 56 49'28" $\mathrm{N}, 117^{\circ} 18^{\prime} 18^{\prime \prime} \mathrm{E}$, elev. $1342 \mathrm{~m}$, mixed forest, on E. esorediosa (thallus), 15.06.2015, S. V. Chesnokov (LE 309252); 56³8'02" N, 117¹1'51" E, elev. $1020 \mathrm{~m}$, Larix gmelinii forest, on E. mesomorpha (thallus), 23.06.2015, S. V. Chesnokov (LE 309259d).

Notes - Ascospores (10.3-)11.4-13.6(-14.5) × (5.0-)6.0-7.6(-8.4) $\mu \mathrm{m}, 1 / \mathrm{b}=(1.5-) 1.6-2.0(-2.2)$ (n=32).

Bryoria simplicior is a new host species.

*Lichenostigma RUPicolae Fern.-Brime \& Nav.Ros. - Headwaters of Olenii Rog Creek, 56 $48^{\prime} 20^{\prime \prime}$ N, 117 $25^{\prime} 17^{\prime \prime}$ E, elev. 2020 m, mountain tundra, on Pertusaria lactea (thallus), 17.06.2015, S. V. Chesnokov (LE 309263c).

Notes - The species was previously known only 
from Spain (Etayo, 2010; Fernandez-Brime et al., 2010) and Russian Caucasus (Zhurbenko \& Kobzeva, 2014). New to Siberia.

*Llimoniella-like discomycete (Fig. 2) - Confluence of Sul'ban River and Zolotoi Creek, 56 $50^{\prime} 13^{\prime \prime} \mathrm{N}, 117^{\circ} 17^{\prime} 24^{\prime \prime}$ E, elev. $1604 \mathrm{~m}$, subalpine Pinus pumila shrubs with stones, on Protoblastenia terricola (thallus), 14.06.2015, L. A. Konoreva (LE 309323).

Notes - Apothecia more or less roundish, up to $0.6 \mathrm{~mm}$ diam., sessile, slightly constricted at the base, shiny, disc plane, brownish black, margin slightly prominent, blackish, dispersed to contiguous by a few. Exciple medium reddish brown/dark reddish orange, $\mathrm{K}+$ brownish orange (red tinge disappears), laterally with darker radial stripes, formed of rarely branched, radiating hyphae with strongly elongate lumina, 40-100 $\mu \mathrm{m}$ thick, basally formed of thick-walled, \pm isodiametric cells mainly $4-8 \mu \mathrm{m}$ diam., 100-200 $\mu \mathrm{m}$ thick, prolonged into an indistinct 'stipe'. Epihymenium pale to medium brownish orange, $\mathrm{K}-$, pigmentation amorphous, up to 10 $\mu \mathrm{m}$ tall. Hymenium colourless, not inspersed, 50-80 $\mu \mathrm{m}$ tall, I-, K/I- except periascal gel (see below). Subhymenium pale to medium brownish

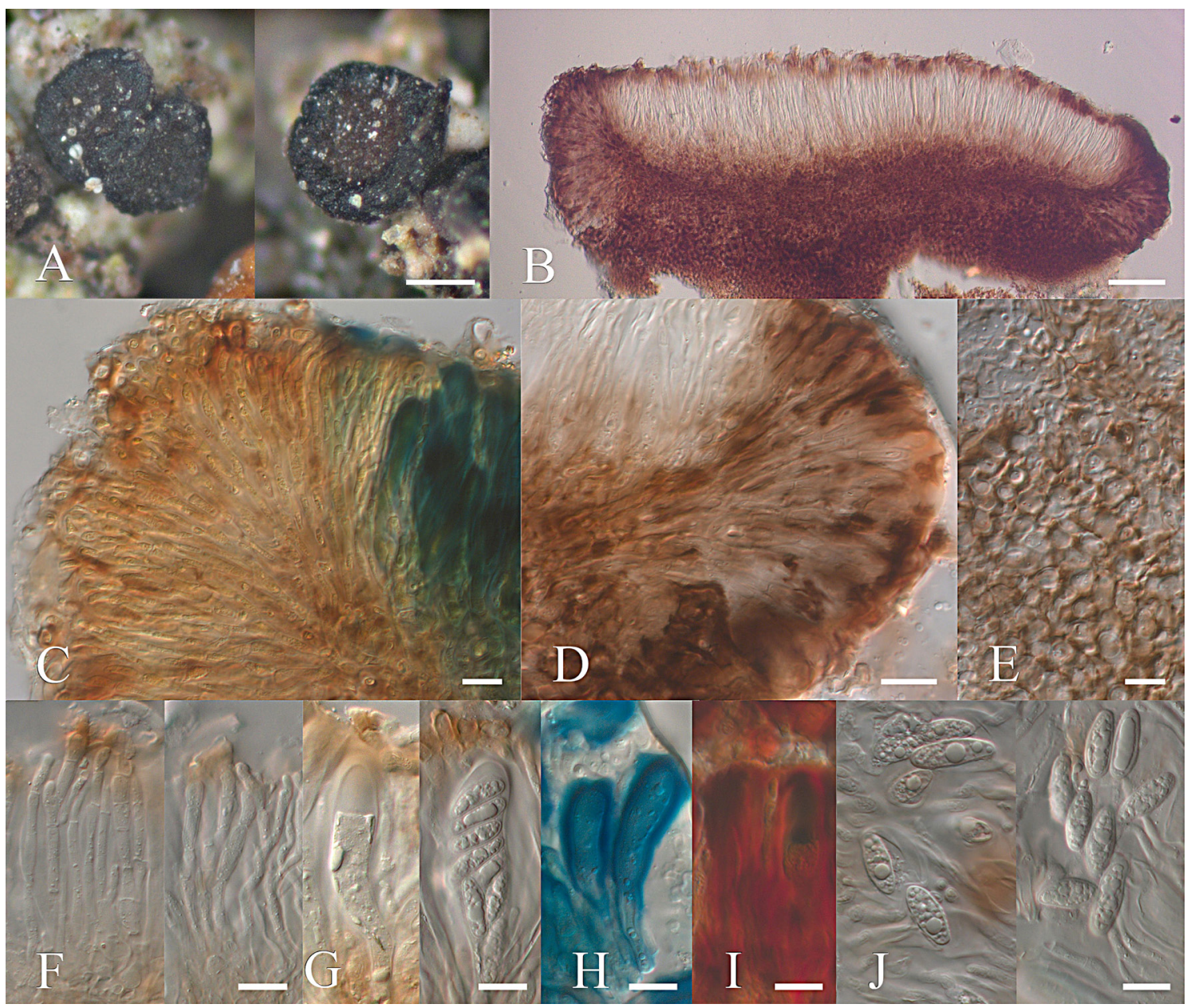

Fig. 2. Llimoniella-like discomycete on Protoblastenia terricola (LE 309323). A - habitus. B - ascoma in section (in water). C - lateral exciple in section (in $\mathrm{K} / \mathrm{I}$ ). D - lateral exciple in section (in $\mathrm{K}$ ). $\mathrm{E}$ - basal exciple in section (in K). F - paraphyses (in K). G - immature (left) and mature (right) asci (in $\mathrm{K}$ ). $\mathrm{H}$ - asci (in $\mathrm{K} / \mathrm{I}$ ). I - asci (in I). $\mathrm{J}$ - ascospores [in water (left) and $\mathrm{K}$ (right)]. Bars: $\mathrm{A}=$ $200 \mu \mathrm{m} ; \mathrm{B}=50 \mu \mathrm{m} ; \mathrm{C}-\mathrm{J}=10 \mu \mathrm{m}$. 
orange, rather indistinct. Paraphyses 2-3(-4) $\mu \mathrm{m}$ diam., septate, usually slightly constricted at the septa and slightly inflated between them, occasionally branched, apically often somewhat swollen, 3-4.5(-5) $\mu \mathrm{m}$ diam., easily separating in $\mathrm{K}$ under slight pressure. Asci unitunicate, nonfissitunicate, narrowly clavate, with rounded apex and long foot, wall apically thickened, up to $15 \mu \mathrm{m}$ thick in immature asci, without ocular chamber, but occasionally with a thin short axial canal, ca. 50-65 × 10-17 $\mu \mathrm{m}, 8$-spored, wall/periascal gel $\mathrm{I}+$ red (sometimes blue in the apical thickening), $\mathrm{K} / \mathrm{I}+$ blue (darker in the apical thickening). Ascospores colourless, narrowly ellipsoid or occasionally oblong, (12.5) 13.8-17.0(-21.0) × (4.9-)5.1-6.3(-7.5) $\mu \mathrm{m}, 1 / \mathrm{b}$ $=(2.0-) 2.4-3.0(-3.6)(\mathrm{n}=66)$, aseptate, usually with one large and/or many small guttules, wall thin, smooth, without perispore, overlappingly biseriate or partly diagonally uniseriate in the ascus. Conidiomata not found. Pathogenicity not seen.

This possibly undescribed fungus is reminiscent of some species of Llimoniella s. 1., which mainly differ in having asci I and K/I-, with only occasionally slightly thickened apical wall (Diederich \& Etayo, 2000; Diederich et al., 2010).

*Merismatium Decolorans (Arnold) Triebel Shan'go Lake, 56 58'45" N, 11748'13" E, elev. 1944 m, mountain tundra, on Peltigera leucophlebia (old discoloured parts of thalli), 7.06.2014, S. V. Chesnokov (LE 309238).

*Muellerella erratica (A. Massal.) Hafellner \& V. John s. 1. - Maloe Leprindo Lake, 56 38'12" N, $117^{\circ} 23^{\prime} 48^{\prime \prime}$ E, elev. $1232 \mathrm{~m}$, rocks, on Lecanora campestris (apothecia, thallus), 17.08.2012, L. A. Konoreva [LE 309246b; ascospores $(5.5-) 6.2-7.8(-9.0) \times(3.5-) 3.6-4.4(-5.1) \mu \mathrm{m}$, $1 / \mathrm{b}=(1.4-) 1.5-1.9(-2.3)(\mathrm{n}=42)]$; confluence of Medvezhii and Surpriznyi Creeks, 56 $54^{\circ} 54^{\prime \prime}$ N, $117^{\circ} 37^{\prime} 41^{\prime \prime}$ E, elev. $1678 \mathrm{~m}$, rocks, on Pleopsidium chlorophanum (thallus), 1.07.2013, M. P. Andreev (LE 309249); Shan'go River, 5657'31" $\mathrm{N}, 117^{\circ} 48^{\prime} 05^{\prime \prime} \mathrm{E}$, elev. $1740 \mathrm{~m}$, stone rubble, on Rusavskia elegans (apothecia, thallus), 9.06.2014, S. V. Chesnokov [LE 309240; ascospores (6.4-)7.0-9.0(-10.8) × (2.8-)2.9-3.5(-3.7) $\mu \mathrm{m}, 1 / \mathrm{b}=(1.9-) 2.1-2.9(-3.9)(\mathrm{n}=25)]$; confluence of Uglovoi Creek and Srednii Sakukan River, 56 56'33" N, $117^{\circ} 36^{\prime} 51^{\prime \prime}$ E, elev. 1670 $\mathrm{m}$, rocky outcrops, on $P$. chlorophanum (thallus), 15.06.2014, S. V. Chesnokov (LE 309255);
Zolotoi Creek, 5650'22" N, $117^{\circ} 18^{\prime} 27^{\prime \prime}$ E, elev. $1407 \mathrm{~m}$, limestones, on Porpidia flavicunda (thallus), 12.06.2015, L. A. Konoreva [LE 309266; ascospores (5.6-)6.4-7.6(-8.9) $\times$ (3.9-)4.2-4.6(-4.9) $\mu \mathrm{m}, 1 / \mathrm{b}=(1.3-) 1.4-1.8(-2.0)$ ( $\mathrm{n}=43)]$.

Notes - The examined specimens significantly vary in the ascospore size. The identification of specimens on Pleopsidium [ascospores $(6.0-) 6.7-8.0(-11.5) \times(3.6-) 3.8-4.6(-5.0) \mu \mathrm{m}$, $1 / \mathrm{b}=(1.3-) 1.6-2.2(-2.9)(\mathrm{n}=47)]$ is somewhat uncertain due to unusual host genus.

*Muellerella lichenicola (Sommerf.) D. Hawksw. - Confluence of Syul'ban and Pravyi Syul'ban Rivers, 5651'37" N, 117²17'22" E, elev. 1420 m, scree, on Lecanora cf. intricata (apothecia, thallus), 11.06.2015, L. A. Konoreva (LE 309278).

Note - New to South Siberia.

Muellerella ventosicola (Mudd) D. Hawksw. - Leprindinskoe Plateau, 56 39'27" N, $117^{\circ} 25^{\prime} 52^{\prime \prime} \mathrm{E}$, elev. $1738 \mathrm{~m}$, mountain tundra, on Immersaria athroocarpa (apothecia, thallus), 16.08.2012, M. P. Andreev (LE 309264; identification uncertain due to unusual host);

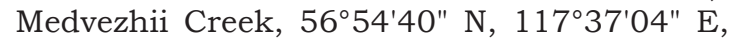
elev. $1764 \mathrm{~m}$, mountain tundra, on Rhizocarpon geographicum (thallus), 1.07.2013, L. A. Konoreva (LE 309270); headwaters of Shan'go River, 56 $58^{\prime} 36^{\prime \prime} \mathrm{N}, 117^{\circ} 48^{\prime} 24^{\prime \prime}$ E, elev. 1874 $\mathrm{m}$, boulders, on Ophioparma ventosa (thallus), 7.06.2014, L. A. Konoreva (LE 309326); Shan'go Lake, 5658'41" N, $117^{\circ} 47^{\prime} 50^{\prime \prime}$ E, elev. 1908 $\mathrm{m}$, mountain tundra, on O. ventosa (thallus), 7.06.2014, S. V. Chesnokov (LE 309320).

*Neolamya Peltigerae (Mont.) Theiss. \& Syd. - Olenii Rog Creek, 5648'14" N, 117²4'16" E, elev. $1860 \mathrm{~m}$, limestones, on Peltigera lepidophora (thallus), 16.06.2015, S. V. Chesnokov (LE 309352).

*Nesolechia fusca (Triebel \& Rambold) PérezOrtega - Shan'go Lake, 56 58'45" N, 117 $48^{\circ} 13^{\prime \prime}$ E, elev. 1944 m, mountain tundra, on Xanthoparmelia stenophylla (thallus), 7.06.2014, L. A. Konoreva (LE 309254a).

Nesolechia oxyspora (Tul.) A. Massal. - Anarga River, 56 55'11" N, $118^{\circ} 01^{\prime} 45^{\prime \prime}$ E, elev. $941 \mathrm{~m}$, mixed forest, on Parmelia sulcata (thallus), 9.07.2013, L. A. Konoreva (LE 309221); Syul'ban River, 56 50'41" N, $117^{\circ} 17^{\prime} 57^{\prime \prime}$ E, elev. $1381 \mathrm{~m}$, Chosenia arbutifolia forest, on $P$. sulcata (thal- 
lus), 13.06.2015, S. V. Chesnokov (LE 309322b); $56^{\circ} 49^{\prime} 28^{\prime \prime} \mathrm{N}, 117^{\circ} 18^{\prime} 18^{\prime \prime}$ E, elev. $1342 \mathrm{~m}$, mixed forest, on $P$. sulcata (thallus), 15.06.2015, S. V. Chesnokov (LE 309253).

*Opegrapha geographicola (Arnold) Hafellner Headwaters of Olenii Rog Creek, 56 $48^{\prime} 31^{\prime \prime}$ N, $117^{\circ} 24^{\prime} 53^{\prime \prime}$ E, elev. $1971 \mathrm{~m}$, mountain tundra, on Rhizocarpon sp. (thallus), 16.06.2015, L. A. Konoreva (LE 309269).

Notes - Formerly known in Russia only from Chukotka (Zhurbenko, 2009b). New to Siberia.

Phacopsis cephalodioides (Nyl.) Triebel \& Rambold - Shan'go River, 5656'22" N, 11748'34" E, elev. $1710 \mathrm{~m}$, mixed forest, on Hypogymnia bitteri (thallus), 10.06.2014, L. A. Konoreva (LE 309330); Syul'ban River, 56²9'28" N, $117^{\circ} 18^{\prime} 18^{\prime \prime} \mathrm{E}$, elev. $1342 \mathrm{~m}$, mixed forest, on $H$. bitteri (thallus), 15.06.2015, S. V. Chesnokov (LE 309251, LE 309257a).

*Phaeospora CF. Rimosicola (Mudd) Hepp Headwaters of Olenii Rog Creek, 56 48'20" $\mathrm{N}, 17^{\circ} 25^{\prime} 17^{\prime \prime} \mathrm{E}$, elev. $2020 \mathrm{~m}$, mountain tundra, on Rhizocarpon umbilicatum (thallus), 17.06.2015, S. V. Chesnokov (LE 309248).

Notes - Ascomata black, globose, 100-200 $\mu \mathrm{m}$ diam., with rough surface, slightly protruding to subsessile; wall reddish brown, $\mathrm{K}-$, externally with dark protrusions, in surface view of textura angularis. Hymenial gel I+ red, K/I+ blue. Distinct interascal filaments not observed. Periphyses 1.5-2(-3) $\mu \mathrm{m}$ diam., septate, occasionally branched, not capitate. Asci clavate or sometimes obclavate, ca. 60-70 × 13-25 $\mu \mathrm{m}$, wall apically thickened up to $4 \mu \mathrm{m}$, distinct ocular chamber not observed, 8-spored, I-, K/I-. Ascospores initially hyaline, later pale orange-brown, broadly to narrowly ellipsoid, narrowly obovoid, occasionally fusiform or narrowly oblong (Fig. 3), (11.2-)14.9-18.5(-20.9) × $(5.5-) 6.4-8.2(-8.9) \mu \mathrm{m}, 1 / \mathrm{b}=(1.3-) 2.0-2.6(-3.3)$ ( $\mathrm{n}=106),(0-) 3-$ septate, sometimes slightly or strongly constricted at the septa, wall $0.5-1.3$ $\mu \mathrm{m}$ thick, smooth, usually with large guttule in each cell when young, sometimes with halo up to $2.5 \mu \mathrm{m}$ thick (in water and particularly in $\mathrm{K}$ ), irregularly 2-3-seriate in the ascus. Pathogenicity not observed.

Sérusiaux et al. (1999) compared two species of Phaeospora known from species of Rhizocarpon, viz. P. parasitica (Lönnr.) Arnold, characterized by ascomata $150-225 \mu \mathrm{m}$ diam. and ascospores 18-23 $\times$ 8-11.5 $\mu \mathrm{m}$, and $P$. rimosicola, characterized by ascomata 60-125 $\mu \mathrm{m}$ diam. and ascospores 16-19 × 5-7 $\mu \mathrm{m}$. According to these characteristics the examined material does not match any of these species, though is closer to Phaeospora rimosicola. However, according to Hawksworth (1985) ascospores of Phaeospora rimosicola type are $18-19 \times 6.5-7 \mu \mathrm{m}$, which better fits the examined specimen. The species was previously known in Asia from Turkey (Halici \& Aksoy, 2009) and Chukotka Autonomous Area of Russia (Karatygin et al., 1999). New to South Siberia.

*Plectocarpon melanohaleae Christnach, Ertz \& Diederich (Fig. 4) - Anarga River, 56 55'19" $\mathrm{N}, 118^{\circ} 01^{\prime} 50^{\prime \prime} \mathrm{E}$, elev. $1071 \mathrm{~m}$, mixed forest, on

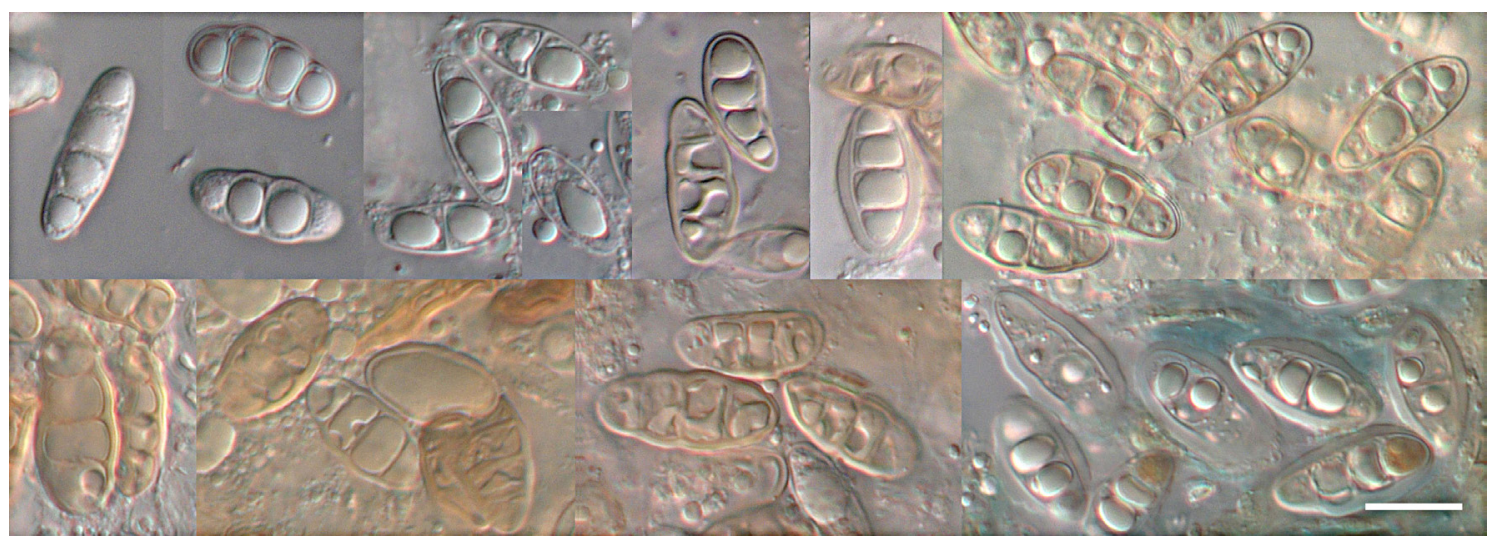

Fig. 3. Ascospores of Phaeospora cf. rimosicola at different stages of maturation (in water, except for the extreme right in the bottom row observed in K/I; LE 309248). Bar $=10 \mu \mathrm{m}$. 


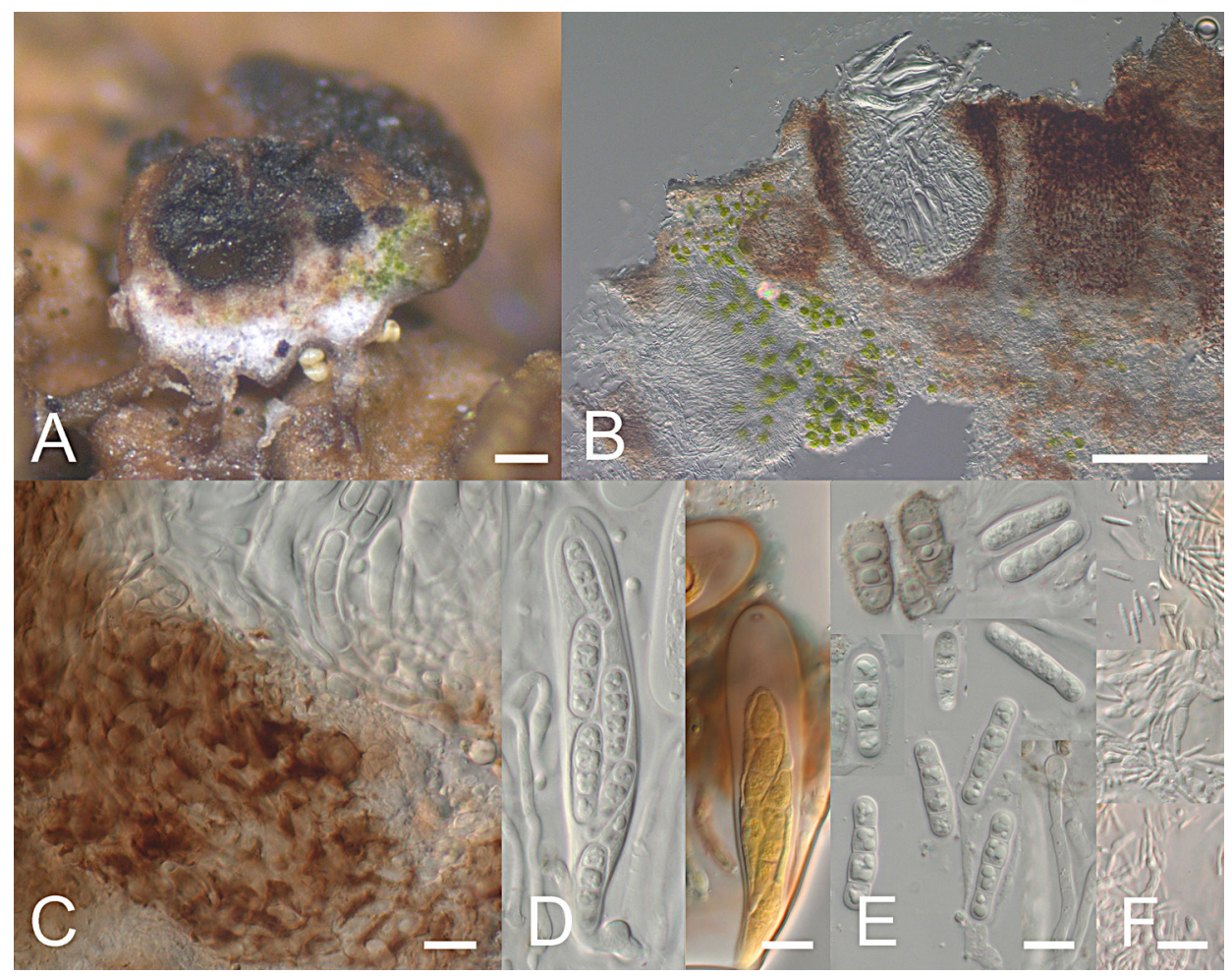

Fig. 4. Plectocarpon melanohaleae (LE 309351c). A - sectioned gall with ascomata. B - ascoma (middle) and conidioma (left) in section (in water). C - stromatic tissue around fertile locule (in water). D - asci [in $\mathrm{K}$ (left) and $\mathrm{K} / \mathrm{I}$ (right)]). $\mathrm{E}$ - ascospores (in K). F - conidiogenous cells and conidia (in K). Bars: $A, B=100 \mu \mathrm{m} ; \mathrm{C}-\mathrm{F}=10 \mu \mathrm{m}$.

Melanohalea olivacea (thallus), 9.07.2013, Yu. V. Gerasimova (LE 309351c).

Notes - Locules subspherical, up to $200 \mu \mathrm{m}$ diam., surrounded by medium to dark reddish brown, $\mathrm{K}+$ gray olive, patchy pigmentated stromatic 'wall'; immersed in lichenized subglobose, constricted below gall $0.7 \mathrm{~mm}$ diam. developed on the host thallus. Hymenium hyaline, I and K/ I+ blue, then quickly red. Paraphyses frequently septate, richly branched, 2-3.5 $\mu \mathrm{m}$ diam., sometimes with enlarged end cells. Asci elongate clavate, $65-100 \times 14-15 \mu \mathrm{m}, 8$-spored, I and $\mathrm{K} / \mathrm{I}-$ except for occasionally observed $\mathrm{K} / \mathrm{I}+$ blue apical ring. Ascospores hyaline, initially with hyaline, smooth perispore, which occasionally becomes brown and granulate at maturity, oblong or slightly tapering below, ends often blunt, (15.5-) 17.6-22.0(-24.4) × (4.3-)4.7-6.3(-7.5) $\mu \mathrm{m}$, $1 / \mathrm{b}=(2.7-) 3.0-4.2(-5.7)(\mathrm{n}=34$, in water, $\mathrm{K}$ or $\mathrm{K} / \mathrm{I}),(1-) 3$-septate, usually slightly constricted at the septa. Conidiomata immersed in stroma between the locules. Conidia hyaline, narrowly fusiform, aseptate, ca. 6-7.5 × $1 \mu \mathrm{m}$.

The studied fungus differs from typical Plectocarpon species, as well as from the species protologue, (Ertz et al., 2005) in looking like aggregated perithecioid ascomata immersed in a bullate gall on the host thallus, instead of multilocular stromatic ascomata. However, it is mentioned in the protologue that the sterile stromatic tissue is poorly developed around fertile locules. The examined material may also represent young ascomata. Another discrepancy with the Plectocarpon concept adopted by Ertz et al. (2005) is a K/I+ red vs. K/I+ blue hymenial gel. Furthermore, in the species protologue conspicuous galls and brown granulate perispore are not mentioned, the paraphyses are described as apically not distinctly enlarged, 1.5-2.5 $\mu \mathrm{m}$ diam., and the asci shorter, 48-70 $\mu \mathrm{m}$ long. So far the species was known only from 
Tierra del Fuego in Chile growing on the thallus of Melanohalea ushuaiensis (Ertz et al., 2005; Etayo \& Sancho, 2008). New for the northern hemisphere. Melanohalea olivacea is a new host species.

*Pronectria Robergei (Mont. \& Desm.) Lowen - Confluence of Syul'ban River and Zolotoi

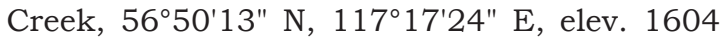
$\mathrm{m}$, subalpine Pinus pumila shrubs, on Peltigera collina (thallus), 14.06.2015, L. A. Konoreva (LE 309241; anamorph).

*Pyrenidium actinellum Nyl. - Headwaters of Shan'go River, 56 58'37" N, 117 49'13" E, elev. 2037 m, mountain tundra, on Baeomyces placophylus (thallus), 8.06.2014, L. A. Konoreva (LE 309367); Uglovoe Lake, 5656'41" N, 117³5'12" E, elev. 1918 m, mountain tundra, on Solorina crocea (moribund thallus), 17.06.2014, S. V. Chesnokov (LE 309321a).

RAESAENENIA HuUsKoneniI (Räsänen) D. Hawksw., Boluda \& H. Lindgr. - Syul'ban River, 56 $38^{\prime} 02^{\prime \prime}$ $\mathrm{N}, 117^{\circ} 11^{\prime} 51^{\prime \prime}$ E, elev. $1020 \mathrm{~m}$, Larix gmelinii forest, on Bryoria sp. (thallus), 23.06.2015, S. V. Chesnokov (LE 309258).

*Roselliniella stereOCAUloRum Zhurb., Kukwa \& Oset - Sul'ban River, 56 49' $27^{\prime \prime}$ N, $117^{\circ} 18^{\prime} 12^{\prime \prime}$ E, elev. $1338 \mathrm{~m}$, Chosenia arbutifolia forest, on Stereocaulon rivulorum (phyllocladia), 10.06.2015, L. A. Konoreva (LE 309359).

Note - The species was previously known in Russia from Baikal Siberia and Yakutiya (Zhurbenko et al., 2009).

Rosellinula haplospora (Th. Fr. \& Almq.) R. Sant.

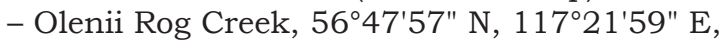
elev. $1674 \mathrm{~m}$, subalpine Pinus pumila shrubs, on gray saxicolous lichen (apothecia, thallus), 18.06.2015, S. V. Chesnokov (LE 309274); on Aspicilia sp. (thallus), 18.06.2015, L. A. Konoreva (LE 309313).

Notes - Ascospores ellipsoid or broadly ellipsoid, mainly with more or less acute apices, occasionally orbicular, medium brown when mature, aseptate, smooth-walled, non-halonate, somewhat smaller than reported by Hafellner (1985), viz. (5.3-)6.3-8.5(-10.4) × (3.9-)4.2-5.2(-6.3) $\mu \mathrm{m}, 1 / \mathrm{b}$ $=(1.0-) 1.3-1.9(-2.3)(\mathrm{n}=79)$ vs. $7-11 \times 4.5-6.5$ $\mu \mathrm{m}$, but of the same size as in LE 261058 specimen, which was also reported from Trans-Baikal Territory (Zhurbenko \& Yakovchenko, 2014).
*Sagediopsis aspiciliae (Vain.) Nik. Hoffm. \& Hafellner - Confluence of Medvezhii and Surpriznyi Creeks, 56⒌'54" N, $117^{\circ} 37^{\prime} 41^{\prime \prime}$ E, elev. $1678 \mathrm{~m}$, rocks, on Aspilidea myrinii (thallus, occasionally apothecia), 1.07.2013, M. P. Andreev (LE 309247); headwaters of Olenii Rog Creek, 5648'31" N, $117^{\circ} 24^{\prime} 53^{\prime \prime}$ E, elev. 1971 $\mathrm{m}$, mountain tundra, on Aspicilia sp. (thallus, occasionally apothecia), 16.06.2015, S. V. Chesnokov (LE 309245); same creek, 56² $8^{\prime} 20^{\prime \prime} \mathrm{N}$, $117^{\circ} 25^{\prime} 17^{\prime \prime}$ E, elev. $2020 \mathrm{~m}$, mountain tundra, on A. myrinii (thallus), 17.06.2015, S. V. Chesnokov (LE 309338a).

Notes - Ascospores (8.5-)9.7-13.7(-17.2) $\times$ $(4.8-) 5.4-7.0(-8.4) \mu \mathrm{m}, 1 / \mathrm{b}=(1.1-) 1.4-2.4(-3.2)$ (n $=34)$. New to South Siberia.

*SAgEdiopsis fissurisedens Hafellner - Zolotoi Creek, 5655'57" N, $117^{\circ} 36^{\prime} 51^{\prime \prime}$ E, elev. 1857 m, mountain tundra, on Aspicilia sp. (thallus), 6.07.2013, L. A. Konoreva (LE 309273).

Notes - Hymenial gel I-, K/I+ blue. Ascospores hyaline, ellipsoid, narrowly ellipsoid, occasionally oblong or slightly obovate, (8.2-)11.2-14.4($15.5) \times(4.1-) 4.3-5.5(-6.5) \mu \mathrm{m}, 1 / \mathrm{b}=(1.8-) 2.1-$ 3.1(-3.6) ( $\mathrm{n}=32), 1-3$-septate, only exceptionally slightly constricted at the septa, usually with a large guttule in each cell, smooth-walled, halo not observed.

In the species protologue the ascospores were reported as ellipsoid, 12-14.5-17 × 5-8 $\mu \mathrm{m}$ (Hafellner, 1993). New to Russia and Asia.

*SAgediopsis Pertusarilcola Zhurb. - Azarova glacier, 5653'58" N, 117³4'59" E, elev. 2053 $\mathrm{m}$, mountain tundra, on Pertusaria-like sterile lichen growing over moribund mosses, 13.06.2014, L. A. Konoreva (LE 309337).

Notes - Formerly known only from the Arctic (Zhurbenko, 2009c). New to South Siberia.

*Sphaerellothecium contextum Triebel - Confluence of Medvezhii and Surpriznyi Creeks, 56 $54^{\circ} 54^{\prime \prime} \mathrm{N}, 117^{\circ} 37^{\prime} 41^{\prime \prime} \mathrm{E}$, elev. $1678 \mathrm{~m}$, rocks, on Calvitimela armeniaca (thallus), 1.07.2013, M. P. Andreev (LE 309279).

Note - New to South Siberia.

*Sphaerellothecium CF. Parmeliae Diederich \& Etayo - Headwaters of Shan'go River, 56 $58^{\prime} 36^{\prime \prime}$ N, 117 49'12" E, elev. 1994 m, mountain tundra, on Parmelia omphalodes (thallus), 8.06.2014, S. V. Chesnokov (LE 309225). 
Notes - The examined specimen is conspecific with those published under the same name (also with 'cf'.) in Zhurbenko \& Zheludeva (2015). This material differs from the species protologue (Etayo \& Diederich, 1998) in that it is not associated with black necrotic areas of the host lobes as well as in several other features, which were noted in the above-mentioned publication.

*Stigmidium conspurcans (Th. Fr.) Triebel \& R. Sant. - Shan'go Lake, 56 58'45" N, 117 $48^{\circ} 13^{\prime \prime}$ E, elev. $1944 \mathrm{~m}$, rocks, on Psora rubiformis (squamules), 7.06.2014, L. A. Konoreva (LE 309224); Olenii Rog Creek, 5648'22" N, $117^{\circ} 23^{\prime} 26^{\prime \prime}$ E, elev. $1874 \mathrm{~m}$, rocks, on P. rubiformis (squamules), 19.06.2015, S. V. Chesnokov (LE 309223).

Notes - Ascospores mostly hyaline and smooth, occasionally pale brown and verruculose when overmature, (11.1-)12.7-15.1(-17.1) × (4.7-)5.06.2(-6.9) $\mu \mathrm{m}, 1 / \mathrm{b}=(1.8-) 2.1-2.9(-3.5)(\mathrm{n}=31$, in water or $\mathrm{BCr}$ ), $\mathrm{BCr}-$. New to South Siberia.

*STigmidium mitchelli Cl. Roux \& Bricaud - Az-

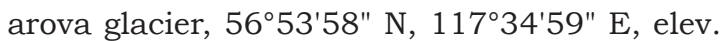
2053 m, mountain tundra, on Protopannaria pezizoides (mostly blackened moribund apothecia, occasionally thallus), 13.06.2014, S. V. Chesnokov (LE 309317).

Note - New to South Siberia.

Stigmidium PSEUdopeltideae Cl. Roux \& Triebel Shan'go River, 56 56'22" N, 117 $48^{\circ} 43^{\prime \prime}$ E, elev. $1841 \mathrm{~m}$, subalpine Betula thin forest, on Peltigera venosa (thallus), 10.06.2014, L. A. Konoreva (LE 309341b).

*Stigmidium pumilum (Lettau) Matzer \& Hafellner - Shan'go River, 56 57'31" N, 117 ${ }^{\circ} 48^{\prime} 05^{\prime \prime}$ E, elev. $1740 \mathrm{~m}$, stone field, on Physcia caesia (thallus), 9.06.2014, L. A. Konoreva (LE 309333); headwaters of Olenii Rog Creek, 56 $48^{\prime} 31^{\prime \prime} \mathrm{N}$, $117^{\circ} 24^{\prime} 53^{\prime \prime}$ E, elev. $1971 \mathrm{~m}$, mountain tundra, on P. phaea (thallus), 16.06.2015, L. A. Konoreva (LE 309261); 56²7'57" N, 117 $21^{\circ} 1^{\prime} 59^{\prime \prime}$ E, elev. $1674 \mathrm{~m}$, subalpine Pinus pumila shrubs, on $P$. caesia (thallus), 18.06.2015, L. A. Konoreva (LE 309327);

*Stigmidium solorinarium (Vain.) D. Hawksw. -

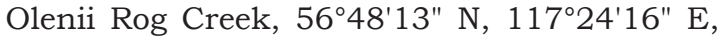
elev. $1827 \mathrm{~m}$, mountain tundra, on Solorina bispora (thallus), 16.06.2015, L. A. Konoreva (LE 309260).

Note - New to South Siberia.
*Taeniolella Beschiana Diederich - Olenii Rog

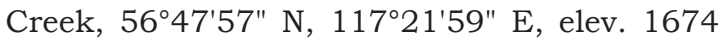
$\mathrm{m}$, subalpine Pinus pumila shrubs, on Cladonia sp. (basal squamules, podetia), 18.06.2015, L. A. Konoreva (LE 309332c).

*Talpapellis peltigerae Alstrup \& M.S. Cole var. PELTIGERAE - Shan'go River, 56 56'22" N, $117^{\circ} 48^{\prime} 43^{\prime \prime}$ E, elev. $1841 \mathrm{~m}$, subalpine Betula thin forest, on Peltigera venosa (thallus), 10.06.2014, L. A. Konoreva (LE 309341a).

Notes - The species was recently revised by Heuchert et al. (2014). It is new to South Siberia, and var. peltigerae is new to Russia and Asia.

"Tetramelas PhaEOPhysciae A. Nordin \& Tibell Zolotoi Creek, 56 55'57" N, 117 $37^{\circ} 6^{\prime} 51^{\prime \prime}$ E, elev. $1857 \mathrm{~m}$, mountain tundra, on Phaeophyscia sciastra (thallus), 6.07.2013, L. A. Konoreva (LE 309239).

Note - According to Nordin \& Tibell (2005) this is an obligately lichenicolous endoparasitic lichen, however, it is often listed together with lichenicolous fungi.

*Unguiculariopsis lettaui (Grummann) Coppins

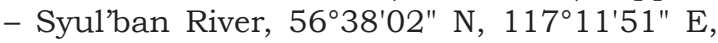
elev. 1020 m, Larix gmelinii forest, on Everina mesomorpha (thallus), 23.06.2015, S. V. Chesnokov (LE 309259a).

Note - Formerly known in Russia only from the Caucasus (Zhurbenko \& Otte, 2012), new to Siberia.

\section{ACKNOWLEDGEMENTS}

Paul Diederich and Javier Etayo provided valuable comments on critical materials. Yu. V. Gerasimova and M. P. Andreev are thanked for putting their lichen specimens at the authors' disposal. The study of M. P. Zhurbenko was carried out within the framework of the research project of the Komarov Botanical Institute, Russian Academy of Sciences (St.-Petersburg, Russia) no. 01201255604 using equipment of its Core Facility Center 'Cell and Molecular Technologies in Plant Science'. The study of S. V. Chesnokov and L. A. Konoreva was financially supported by the Russian Foundation for Basic Research (grants 14-04-01411, 15-04-05971). 


\section{REFERENCES}

Alstrup, V. \& Ahti, T. 2007. New reports of lichenicolous fungi, mainly from Finland and Russia. Karstenia 47: 1-4.

Alstrup, V., Christensen, S. N., Hansen, E. S. \& Svane, S. 1994. The lichens of the Faroes. Fródskaparrit 40: 61-121.

Berger, F., Priemetzhofer, F. \& Türk, R. 1998. Neue und seltene Flechten und lichenicole Pilze aus Oberösterreich, Österreich IV. Beitrage Naturkunde Oberösterreichs 6: 397-416.

Boissiere, J. C. \& Montavont, J. P. 1998. Lichens de France (XI): Epigloea filifera Döbb. et Lobaria amplissima (Scop.) Forss. dans sa forme avec céphalodies. Bulletin d'Informations de l'Association Française de Lichénologie 23: 1-6.

Brackel, W. v. 2009. Weitere Funde von flechtenbewohnenden Pilzen in Bayern - Beitrag zu einer Checkliste IV. Berichte der Bayerischen Botanischen Gesellschaft 79: 5-55.

Brackel, W. v. 2010. Some lichenicolous fungi and lichens from Iceland, including Lichenopeltella uncialicola sp. nov. Herzogia 23: 93-109. http:// dx.doi.org/10.13158/heia.23.1.2010.93

Brackel, W. v. 2011. Lichenicolous fungi and lichens from Puglia and Basilicata (southern Italy). Herzogia 24: 65-101. http://dx.doi.org/10.13158/ heia.24.1.2011.65

Diederich, P. 2004. Abrothallus. In: T. H. Nash III, B. D. Ryan, P. Diederich, C. Gries and F. Bungartz (eds.). Lichen Flora of the Greater Sonoran Desert Region. Vol. 2. Lichens Unlimited, Arizona State University, Tempe, Arizona. Pp. 626-630.

Diederich, P. \& Etayo, J. 2000. A synopsis of the genera Skyttea, Llimoniella and Rhymbocarpus (lichenicolous Ascomycota, Leotiales). Lichenologist 32: 423-485. http://dx.doi.org/10.1006/ lich.2000.0290

Diederich, P., Ertz, D. \& Etayo, J. 2010. An enlarged concept of Llimoniella (lichenicolous Helotiales), with a revised key to the species and notes on related genera. Lichenologist 42: 253-269. http: / / dx.doi.org/10.1017/S0024282909990612

Döbbeler, P. 1984. Symbiosen zwischen Gallertalgen und Gallertpilzen der Gattung Epigloea (Ascomycetes). Beiheft zur Nova Hedwigia 79: 203-239.

Ertz, D., Christnach, C., Wedin, M. \& Diederich, P. 2005. A world monograph of the genus Plectocarpon (Roccellaceae, Arthoniales). Bibliotheca Lichenologica 91: 1-155.

Ertz, D., Diederich, P., Lawrey, J. D., Berger, F., Freebury, C. E., Coppins, B., Gardiennet, A. \& Hafellner, J. 2015. Phylogenetic insights resolve Dacampiaceae (Pleosporales) as polyphyletic: Didymocyrtis (Pleosporales, Phaeosphaeriaceae) with Phoma-like anamorphs resurrected and segregated from Polycoccum (Trypetheliales, Polycoccaceae fam. nov.). Fungal Diversity 74: 53-89. http:/ /dx.doi.org/ 10.1007/s13225-015-0345-6
Etayo, J. 2010. Líquenes y hongos liquenícolas de Aragón. Guineana 16: 1-501.

Etayo, J. \& Diederich, P. 1998. Lichenicolous fungi from the western Pyrenees, France and Spain. IV. Ascomycetes. Lichenologist 30: 103-120. http:/ / dx.doi.org/10.1017/S0024282992000112

Etayo, J. \& Sancho, L. G. 2008. Hongos liquenícolas del Sur de Sudamérica, especialmente de Isla $\mathrm{Na}-$ varino (Chile). Bibliotheca Lichenologica 98: 1-302.

Fernandez-Brime, S., Llimona, X. \& Navarro-Rosines, P. 2010. Lichenostigma rupicolae (Lichenotheliaceae), a new lichenicolous species growing on Pertusaria rupicola. Lichenologist 42: 241-247. http: / /dx.doi.org/10.1017/S0024282909990727

Hafellner, J. 1985. Studien uber lichenicole Pilze und Flechten III. Die Gattung Roselliniella Vainio emend. Haf. (Ascomycotina, Dothideales). Herzogia 7: 145-162.

Hafellner, J. 1993. Über Funde von lichenicolen Pilzen und Flechten im südlichen Norwegen. Herzogia 9: 749-768.

Hafellner, J. \& Türk, R. 1995. Über Funde lichenicoler Pilze und Flechten im Nationalpark Hohe Tauern (Kärntner Anteil, Österreich). Carinthia II 185/105: 599-635.

Hafellner, J. \& Wieser, B. 2000. Beitrag zur Diversitat von Flechten und lichenicolen Pilzen im oststeirischen Hugelland unter besonderer Berucksichtigung der Gebiete mit anstehenden Vulkaniten (Steiermark, Osterreich). Fritschiana 23: 1-26.

Halici, M. G. \& Aksoy, A. 2009. Lichenised and lichenicolous fungi of Aladağlar National Park (Niğde, Kayseri and Adana Provinces) in Turkey. Turkish Journal of Botany 33: 169-189.

Hawksworth, D. L. 1971. Tichothecium alectoriae sp. nov. on Alectoria ochroleuca. Transactions of the British Mycological Society 57: 338-339. http:/ / dx.doi.org/10.1016/S0007-1536(71)80016-5

Hawksworth, D. L. 1981. The lichenicolous coelomycetes. Bulletin of the British Museum (Natural History), Botany Series 9: 1-98.

Hawksworth, D. L. 1985. A redisposition of the species referred to the ascomycete genus Microthelia. Bulletin of the British Museum (Natural History), Botany Series 14: 43-181.

Heuchert, B., Zhurbenko, M. P. \& Braun, U. 2014. Reassessment of the lichenicolous hyphomycete genus Talpapellis. Herzogia 27: 83-92. http:// dx.doi.org/10.13158/heia.27.1.2014.83

Karatygin, I. V., Nezdoiminogo, E. L., Novozhilov, Y. K. \& Zhurbenko, M. P. 1999. Russian Arctic Fungi. Annotated checklist. St.-Petersburg: Khimikopharmatsevticheskaya Akademiya. 212 p. (In Russian, English summary).

Khodosovtsev, A. Y. 2005. New lichen genera for Ukraine. Ukrainian Botanical Journal 62(2): 170-174. 
Kocourková, J. \& van den Boom, P. P. G. 2005. Lichenicolous fungi from the Czech Republic II. Arthrorhaphis arctoparmeliae spec. nov. and some new records for the country. Herzogia 18: 23-35.

Kulakov, V. S., Ryzhiy, V. S. \& Snegur, A. E. 2002. Geography of Kalarskii District. Chita. 252 p. (In Russian).

Lawrey, J. D. \& Diederich, P. 2016. Lichenicolous fungi - worldwide checklist, including isolated cultures and sequences available. URL: http:// www.lichenicolous.net. (accessed 24.06.2016).

Nordin, A. \& Tibell, L. 2005. Additional species in Tetramelas. Lichenologist 37: 491-498. http:// dx.doi.org/10.1017/S0024282905015434

Orange, A. 2002. Lichenicolous fungi on Ionaspis lacustris. Mycotaxon 81: 265-279.

Räsänen, V. 1939. Die Flechtenflora der nördlichen Küstengegend am Laatokka-See. Annales Botanici Societatis Zoologicae-Botanicae Fennicae 'Vanamo' 12(1): 1-240.

Santesson, R. 1960. Lichenicolous fungi from northern Spain. Svensk Botanisk Tidskrift 54(4): 499-522.

Santesson, R., Moberg, R., Nordin, A., Tønsberg, T. \& Vitikainen, O. 2004. Lichen-forming and lichenicolous fungi of Fennoscandia. Museum of Evolution, Uppsala University. 359 p.

Sérusiaux, E., Diederich, P., Brand, A. M. \& van den Boom, P. 1999. New or interesting lichens and lichenicolous fungi from Belgium and Luxembourg. VIII. Lejeunia N. S. 162: 1-95.

Smith, C. W., Aptroot, A., Coppins, B. J., Fletcher, A., Gilbert, O. L., James, P. W. \& Wolseley, P. A. (eds). 2009. The Lichens of Great Britain and Ireland. British Lichen Society, London. 1046 pp.

Suija, A. 2005. Lichenicolous fungi and lichens in Estonia I. Ascomycota. Nova Hedwigia 80(1-2): 247-267. http://dx.doi.org/10.1127/00295035/2005/0080-0247

Triebel, D. 1989. Lecideicole Ascomyceten. Eine Revision der obligat lichenicolen Ascomyceten auf lecideoiden Flechten. Bibliotheca Lichenologica 35: $1-278$.

Urbanavichus, G. \& Urbanavichene, I. 2011. New records of lichens and lichenicolous fungi from the Ural Mountains, Russia. Folia Cryptogamica Estonica 48: 119-124.

van den Boom, P. 1998. Enkele interessante vondsten van lichenen en lichenicole fungi in Nederland II. Buxbaumiella 47: 40-43.
Zhurbenko, M. P. 2009a. Lichenicolous fungi and some lichens from the Holarctic. Opuscula Philolichenum 6: 87-120.

Zhurbenko, M. P. 2009b. Lichenicolous fungi and lichens from the Holarctic. Part II. Opuscula Philolichenum 7: 121-186.

Zhurbenko, M. 2009c. Sagediopsis pertusariicola (Verrucariales), a new lichenicolous ascomycete from the Arctic. Nova Hedwigia 88(3-4): 549-555. http://dx.doi.org/10.1127/00295035/2009/0088-0549

Zhurbenko, M. P. 2012. Lichenicolous fungi growing on Thamnolia, mainly from the Holarctic, with a worldwide key to the known species. Lichenologist 44: 147-177. http://dx.doi.org/10.1017/ S0024282911000739

Zhurbenko, M. P. \& Davydov, E. A. 2000. Lichenicolous fungi and some lichens from the Russian Altai, southern Siberia. Folia Cryptogamica Estonica 37: 109-118.

Zhurbenko, M. P. \& Kobzeva, A. A. 2014. Lichenicolous fungi from Northwest Caucasus, Russia. Herzogia 27: 377-396. http://dx.doi.org/10.13158/ heia.27.2.2014.377

Zhurbenko, M. P. \& Otnyukova, T. N. 2001. Lichenicolous fungi from the Sayan-Tuva Mountains, Southern Siberia, Russia. Folia Cryptogamica Estonica 38: 79-84.

Zhurbenko, M. P. \& Otte, V. 2012. Lichenicolous fungi from the Caucasus: new records and a first synopsis. Herzogia 25: 235-244. http://dx.doi. org/10.13158/heia.25.2.2010.235

Zhurbenko, M. P. \& Yakovchenko, L. S. 2014. A new species, Sagediopsis vasilyevae, and other lichenicolous fungi from Zabaikal'skii Territory of Russia, southern Siberia. Folia Cryptogamica Estonica 51: 121-130. http:/ /dx.doi.org/10.12697/ fce.2014.51.14

Zhurbenko, M. P. \& Zheludeva, E. V. 2015. Lichenicolous fungi from Russia, mainly from the Magadan Region. Folia Cryptogamica Estonica 52: 101-107. http: / /dx.doi.org/ 10.12697 / fce.2015.52.13

Zhurbenko, M. P., Kukwa, M. \& Oset, M. 2009. Roselliniella stereocaulorum (Sordariales, Ascomycota), a new lichenicolous fungus from the Holarctic. Mycotaxon 109: 323-328. http://dx.doi. org/ 10.5248/109.323 